\title{
A critical evaluation in the delivery of the ultrasound practice: the point of view of the radiologist
}

\author{
Giovanni Stasi, Elsa M. Ruoti \\ Radiology Department, Villa Chiara, Casalecchio di Reno (BO), Italy
}

\begin{abstract}
The authors describe the benefits and risks of massive and widespread diffusion of ultrasound diagnostics for public and affiliated centers. The main considerations regarding organizational aspects, cultural and qualitative assistance of various medical professionals belonging to the Public Service, unfortunately point out that not all lesions found are able to use the same standardized diagnostic criteria. The risks of errors in diagnostic consumerism, with consequent multiplication of exams, often redundant, are on the rise. The authors urge greater control and the adoption of stricter criteria for the selection of clinical skills of operators who are responsible for this delicate, difficult and at the same time valuable diagnostic task, moreover, of great social value.
\end{abstract}

\section{Introduction}

The increase in the supply of ultrasound performance has not recorded any pause in the last decade. Its application has registered a continuous expansion in indications and clinical use. This growth was supported by a consistent and undisputed expansion of its diagnostic possibilities. ${ }^{1}$ However, owing to the development, expansion and upgrading of the diagnostic ultrasound imaging, some organizational considerations are necessary, together with a continuous surveillance, on the technological aspects of the clinical competence and the coordination between ultrasound practitioners and prescribers, in order to achieve more qualified performances, possibly free from waste, errors and malpractice. In this context we have to consider both the criteria on which diagnostic ultrasound imaging examinations are requested, often not only according to

Correspondence: Giovanni Stasi, Radiology Department, Villa Chiara, via Porrettana 170, 40033 Casalecchio di Reno (BO), Italy.

Tel.: +39.051.2984900 - Fax: +39.051.560102.

E-mail: giovstasi@gmail.com

Key words: organization of diagnostic ultrasound, clinical competence of ultrasonologist.

Received for publication: 7 March 2014. Accepted for publication: 7 March 2014

This work is licensed under a Creative Commons Attribution NonCommercial 3.0 License (CC BY-NC 3.0).

(C) Copyright G. Stasi and E.M. Ruoti, 2015

Licensee PAGEPress, Italy

Italian Journal of Medicine 2015; 9:5-10

doi:10.4081/itjm.2015.502 clinical criteria, ${ }^{2}$ both the offer of diagnostic ultrasound imaging, with a detailed survey of the operating standards and technological-organizational conditions of any single center. The comparison of the diagnostic possibilities among existing imaging techniques and the choice of the most appropriate for any specific case concerns both the prescriber and the sonographic practitioner, having more and more numerous and promising available alternatives. A correct selection in the diagnostic procedures has some obvious advantages such as reducing delays, countering health consumerism, avoiding the fragmentation of care and standardizing their indications..$^{3-7}$ For these goals, it is important to define in the different health care settings shared guidelines and clinical pathways, mainly for the most common and economically burdensome diseases, whilst respecting the prescriptive discretion and autonomy of professionals. The wide range of health professionals that may require an ultrasound test (for example, a general practitioner, internist, surgeon, neurologist, dermatologist, etc.) may generate unsuitable prescription when not consistent with the diagnostic shared algorithms.

\section{Organizational and competence related issues are important in the implementation of an ultrasound service}

The continuous proliferation of new centers and delivery points of ultrasound, not always controlled as standardized quality requirements, requires a discussion about some organizational and cultural issues, also to avoid risks connected with malpractice and errors. In the development, accreditation of new operators and increasing workload of a center of echography different issues, often underestimated by professionals, need to be considered (Table 1). 
In addition to these important organizational aspects, we need to consider some issues related to the competence of professionals, which makes the effectiveness of ultrasonography operator dependent (Table 2).

\section{Which competence is required in the current organization of the ultrasound practice?}

In the public and private hospital setting, the cultural background of the pure ultrasound Practitioner is a guarantee of competence, providing a common understanding, over-specialized vision, across several delivery points, able to deal with diseases of each organ and system, with a diagnostic continuity $24 \mathrm{~h}$ a day, throughout the year. Without detracting from the excellent skills and performances of the neo-specialist ultrasound doctors from different backgrounds (designed to provide more specific tests of second level) we believe that the specialist who can provide all this is the radiologist. He can ensure the use of different methods (conventional radiology, conventional echo and/or by contrast agent, computed tomography, magnetic resonance imaging, etc.), all performed from the same professional at the same time. The principle of the diagnostic-therapeutic continuity, preferably provided by a single operator or by the same team, is sanctioned by precise rules and should be pursued whenever possible. Obviously, this perspective implies that professionals are competent, motivated, updated and with a sub-specialized and trans-professional vocation. The positive aspects of this organization, which recognizes a homogeneous and constant relationship between the issuing institution and the individual patient, are important; they could be briefly summarized as follows: i) the implementation of the continuity of diagnosis able to guarantee homogeneous criteria in the evaluation of the lesions observed; ii) the highest comprehensiveness of the diagnostic act, including further investigations, such as miniendoscopic (for example trans-vaginal ultrasound) or mini invasive (as ultrasound-guided fine needle aspiration or biopsy); iii) centralization of the diagnostic procedures in accredited structures; iv) the opportunity of direct relations between referring physicians and sonographers, who can be contacted in every single Operating Unit. Any organizational solution, open to new aspirant ultrasound practitioner clinicians, could imply a dispersion of the activity, with some disadvantages, highlighted in Table 3.

It could be argued that the topic is outdated. In

Table 1. Some organizational issues to be considered in the exercise of the ultrasonodiagnostic activity.

The excessive fragmentation of the ultrasound diagnostic act, often isolated and not integrated with the results of complementary methods, previous or successive, whose the operator, often, is unaware

Potential lack of appropriate ways in the subsequent reviews of the lesions, especially with regard to the seat, the timing and the type of the technique used in the following controls, after the first ultrasound finding

Evolutionary lesions should be re-checked in the same setting where they were found the first time, by the same professionals just to ensure the control reliability and the continuity between patients, prescribers and sonographer practitioners

The different instrumental equipment in individual centers, their quality, age and obsolescence

The complexity of some ultrasound examinations, contrasting with the excessive indefiniteness of the nomenclature of the examinations acquired by the booking centers, moreover not always aware of the specific expertise of the operators; this may lead to excessive delays for some difficult or challenging examinations (e.g., intracavitary ultrasound, echo-color-Doppler of parenchymatous organs, soft tissues ultrasonography, etc.) and therefore not available in the short term, resulting in disparities between different investigation surveys of waiting times

The absence of shared required standard criteria of quality of iconographic documentation, in archiving and de-archiving practices, in referring the patient's history in the medical reports, in indicating additional examinations, if indicated

Table 2. Clinical competence and skills have to be considered in the exercise of the ultrasonographic activity.

The variability in different professional extraction of sonographers, consisting of experts from different backgrounds (generalists, internists, radiologists, surgeons, etc.) can lead to possible differences in the interpretation the ultrasonographic pathological alterations of the same organ in different settings

Such variability may affect a different diagnostic behavior in the subsequent phases, with choices sometimes very discretional, even in the presence of the same pathology

The absence of a stable use over the time of shared interpretative criteria of lesions with respect to the progressive enrichment of ultrasound semeiotic patterns and the introduction of new methods and diagnostic tests

The tout court individual operator confidence about diagnostic ultrasound and the most recent methods (color-Doppler, 3D and 4D ultrasound, etc.) can lead to self-referential not certified credits 
Italy the ultrasound practice has been granted to everyone, because lacking of rules is limiting its potential users. Moreover, many best Italian ultrasound practitioners include non-radiologists. Indeed in the health care world we observe somewhat of everything. Someone expects an expansion to other categories of users of ultrasound practice; up to include the professional nurses, as for the diagnostic medical sonographers in the United States. On the other hand, there are radiologists who refuse to deal with ultrasound, because they prefer to practice other procedures, such as interventional radiology, nuclear magnetic resonance and so on. There are general doctors who have an ultrasound appliance in their office and they perform the ultrasound directly. On the basis of these considerations, the key message that we would like to introduce is to invest in experienced operators, properly trained and able to use the principles of the clinical audit and management control and to check the results of their diagnosis.

\section{The general practitioner's and generalist's ultrasound activity}

The opportunity to extend the diagnostic ultrasound to general practitioners (GPs) is well established. ${ }^{8}$ However, the use of ultrasound by GPs in their clinic, even if in many ways commendable, has some limitations: for example, in everyday medicine, with respect to the complexity of the presentation of some diseases, often oligosymptomatic, sometimes witnessed by vague symptoms, nonspecific, ambiguous, misleading, not always attributable to a specific organ, how is it possible to limit or avoid the false negative in the ultrasound diagnostics? From an ethical and social perspective can we admit to skim over potential errors? Is it socially acceptable a false reassurance of a negative ultrasound report, performed by an operator who recognizes his own limits of experience? An answer to this question is given by the Association of Medical Diagnostics Tuscany ${ }^{9}$ with simple and wellargued comments, not agreeing to the idea for which family's doctors could perform some diagnostic ultrasound examinations of $1^{\text {st }}$ level, in order to relieve the pressure on the Emergency Rooms and to reduce the waiting lists. In this respect, some aspects need to be highlighted: i) the so-called $1^{\text {st }}$ level ultrasound is a term increasingly used, but it has never been well defined: for us there is only the ultrasound in the excellence. If a disease is not recognized at the first ultrasonographic examination why should a more experienced operator, with unnecessary waste of time and resources, repeat it? ii) a general practitioner should be trained in ultrasound and authorized to carry out investigations only after evaluating a large number of cases; iii) if a doctor performs an ultrasound, is he required to medical reports? And if he does not have this duty, how is it possible to ensure safety to the patient? Nowadays our colleagues of General Medicine are overworked by heavy workloads. How will they find the time to perform an ultrasound examination when not completely experienced to prepare the related medical report?

Table 3. Some disadvantages related to an excessive dispersion of the ultrasounds delivery points.

Extension like wild fire of requests for authorization to perform ultrasound examinations by other physicians

The potential (and perhaps dangerous) small number of treated cases in peripheral OU

The need to lead to training at least three operators for each OU

The risk of errors or of over diagnosis of elementary lesions at the first ultrasound examination, with consequent induction to further investigation, not risk-free, and which may also increase the length in hospital stay: it is not uncommon that radiological or endoscopic surveys take place only to reassure the physician on the patient's health, threatened by an initial sonographic misdiagnosis by a misguided neo-sonographer

The need of additional administrative assistance

Subtraction of time job assistance and reduction of production

Recruitment of pathologists of higher educational value, but with a limited variability, when studying only few diseases

This exclusive management by clinical ultrasound practitioner increases the risk of dispersion of the cases, removing them from the expertise and professional development of other ultrasound practitioners

The case of segmentation of skills may lead to an abuse in the request of a second opinion (e.g. the study of a pelvic mass or of a superficial lesion managed by an ultrasound practitioner of internist extraction could imply a need of the repetition of the same examination from another more experienced doctor)

The need to arrange new channels of follow-up: this can hardly be guaranteed by the sonographer clinician

The complexity in inclusion, often owing to their excessive sector-based specialization, of clinical ultrasound practitioner on turns on-call of the doctors who perform activities eco-diagnostic (why should this duty fall back on only the availability of radiologists?)

OU, Operating Unit. 


\section{Echo-focused assessment with sonography for trauma}

The so-called focused assessment with sonography for trauma (FAST) is a rapid (mostly focused than faster!) ultrasound examination performed in an emergency situation by an operator that is not used to perform a discipline of imaging (often an emergency physician) or by some other health care professional (who is not a physician), in order to exclude a posttraumatic blood extravasation in the pericardial or abdominal. ${ }^{10,11}$ The four classical anatomical areas explored in search of a possible fluid collection, are: the hepatorenal Morison's pouch, the perisplenic lodge, the pericardium and the pelvic region. The reliability of this method, however, is not very high, especially in trauma. Some contributions show how the repetition of ultrasound, focused to critical anatomical areas, or the integration with other methods, are adding accuracy to studies, recovering the false-negative escaped from FAST-echo. ${ }^{12,13}$

\section{Ultrasound related risks and pitfalls in the diagnostic process}

Bedside ultrasound should always be used in the context of the clinical scenario and should never replace the physical exam. In the ultrasound practice, as in direct care of the patient, two basic error types can occur: errors of ignorance and errors of implementation. Errors of ignorance are due to inadequate knowledge, whereas errors of implementation occur during application of knowledge. ${ }^{14}$ The percentage of errors in ultrasound diagnostics is similar to that of other medical disciplines (Ialongo M, personal communication, 2000). It is unchanged over the last years, despite the significant increase in its use. Ultrasound quality is operator dependent and subjective to interpretive error: in successfully integrating this technology into their clinical practices, physicians must be familiar with the normal and abnormal appearance of tissues. They must also recognize the clinically relevant limitations and pitfalls associated with the use of ultrasound. This phenomenon cannot be attributed only to the false negatives. It may depend on several other items, such as the lack of a methodologically correct clinical approach, excessive diagnostic confidence, generated by superficiality and/or lack of experience. Many of some diagnostic inaccuracy cases are represented by the late acquisition of information or facts from the collection of the history and the consequent formulation of the clinical question. In the error's evaluation we have to discriminate mistakes attributable to the individual from those due to whole organization. Individual practitioner's mistakes may be attributable to the lack of rigorous clinical method or to carelessness, forgetfulness or neglect, in addition to potential existing technological deficiencies. Medical diagnosis is a cognitive process, which is carried out through two methods, one of inductive type, the other one deductive. The inductive cognitive method used in the activity diagnostics is empirical, based on a finite number of observations of a given phenomenon, formulating hypotheses throughout the use of a cognitive process of heuristic type. It's faster, similar to the skills that we need for driving a car. This process identifies any new situation as similar examples already experienced in our memory whose recovery is rapid and almost automatic. The second system, slower than the first one is deductive, generally considered more effective, because analytical, on logical and rational rules based. Both methods, considered separately, are subjected to errors, but if reciprocally integrated, they can achieve an optimal outcome. The inductive system, rapid and automatic, it is often the first to come into operation during a cognitive process, the task of the deductive system is to modulate the first by limiting the errors. Our level of knowledge and the unaware memory of the experienced work could help in discriminating decisions made using each of the two systems, in order to achieve an optimal result. In the daily practice it is not always easy to handle diagnostic mistakes, the most of these are related to overconfidence. The excessive diagnostic confidence (overconfidence), not supported by a corresponding experience resulting from the treatment of large series, may be one of the causes. ${ }^{15,16}$ Over-confidence is something that could plague all human beings in a variable way, but individuals less competent are those who tend to overestimate their cognitive abilities and then to run into this type of error, dictated often, by an attitude of arrogance (I know all I need to know) ${ }^{17}$ On the contrary, a right behavior should reflect the well-known Socrates's aphorism: I know that I know nothing, implying a concept of ignorance as a continuous stimulus that should lead all of us to improvement of our knowledge. In the context of ultrasonography we should point out the insidiousness of certain images that may cause errors of interpretation, underlying the complexity of this activity and the need for the diagnostician to extract from the images, not just those from ultrasounds, the correct information, avoiding to collect irrelevant or erroneous data. In a wrong interpretation of the images, after highlighting the most important injury at the first examination, is related to a lack of holistic and the panoramic viewing of the display in relation to the nonobservation of ancillary, but relevant concomitant findings concurrent to that mainly detected. In other words, finding the first lesion can run out by itself the initial diagnosis. A hurried and superficial clinician is sometimes inclined to accept what occurred at a first evaluation of the images reflecting his diagnostic 
expectations, contributing in this way, to the failure of the whole diagnostic process. Ashmann et al. ${ }^{18}$ consider equal to $50 \%$ the number of situations in which the second or the third lesion have not been recognized after the detection of the first one. In general, there are four main reasons why radiologists, as professionals, are sued: observer errors, errors in interpretation, failure to suggest the next appropriate procedure and failure to communicate in a timely and clinically appropriate manner. ${ }^{19} \mathrm{~A}$ useful classification of misinterpretation and bad communication related errors of pathological findings in ultrasonographic practice is described in Table 4. It emphasizes the importance of the description of pathological changes detected, which should not be ambiguous, but easy to understand, in the form of detailed reporting, timely with respect to the clinical question.

Some other failures that could be referred to the system or to the organization, belong to the chapter of risk management. These situations can be attributable to administrative factors, mostly related to the bureaucratic procedures and those determined by the health care context and communication system, that does not allow to follow the patient just to the final diagnosis (the so-called non-feedback system). ${ }^{20}$

\section{The importance of clinical reasoning and audit in the ultrasonodiagnostic practice}

In the medical practice, we have to affirm the opportunity to use an old methodology based on the interpretation of a case that should inspire the clinical reasoning. This may be achieved by relying on our proper intuition, recalling similar cases encountered in the past, considering some other different assumptions and alternatives to the first hypothesis (the strategy of considering-the-opposite), even through the help of other colleagues and the consultation of scientific sources. The whole patient pathway should be subject to clinical audit under the categories of structure, process and outcome..$^{21,22}$

\section{Conclusions}

Despite a wider desired spread of ultrasonography in health care settings, the allocative widespread distribution of ultrasonographic equipment has not yet achieved a clear reduction of waiting times, without decreasing the use of other investigations additional alternatives. On the contrary, the increased number of devices and operators devoted to ultrasound caused a multiplication of examinations, instrumental checks, interpretational doubts, with the necessity of more diagnostic confirmations, involving more costly complex examinations and an increased waiting time for the final diagnosis. The price paid to the diffusion and the extensive use in multiple clinical applications, has been very high, moving the ultrasound diagnosis outside of an economic control, without any warranty of the quality of the product, often questionable, depending on different ultrasound delivery centers.

Table 4. Pathological findings at an initial ultrasound diagnosis and the corresponding errors.

Unseen

Invented

Seen, but misinterpreted

Seen, well understood, but poorly described

Seen, well understood, well described, but poorly communicated

Table 5. Some of quality indicators in ultrasound delivery of care.

Annual productivity reporting, as an important indicator of efficiency and clinical experience's operator

Request of additional staff in supporting activities

Expertise areas (whole body or not) and distinctive skills: eco-cardiography, use of contrast, color-Doppler, echo interventional

$24 \mathrm{~h}$ (or not) guarantee of activities

Existence of guidelines, operating rules, algorithms and quality procedures (and their application)

Participation in clinical pathways and audits in the local health care system

Interactive communication with prescribers

Safekeeping, storage and de-archiving ability of the original images in the informative 
Our reflection is not a critique on the work of anyone, still less on those responsible for the allocation of resources. It aims to suggest the adoption of some simple criteria of judgment, concerning the control of management, without any claim to resolve the matter, in a context of organizational and economic uncertainty. Our assessment of the organization of diagnostic ultrasound in the panorama of the public performance is very personal and perhaps too pessimistic, but it predicts the existence of precise quality requirements in each of the distribution centers. This evaluation may help to promote new responsibilities for policy makers, stimulating the acquisition of new knowledge indicators in this field of delivery of care (Table 5).

In our opinion, the coordination of activities should point out, in order to rationalize costs, the reduction of waste, limiting the dispersion and redundancy of the ultrasound delivery points, selecting the accredited centers, defining organizational protocols, looking for a more and more quality health care system.

\section{References}

1. Hall FM. Overutilization of radiological examinations. Radiology 1976;20:443-8.

2. Stasi G. Riflessioni sul governo della domanda in Radiologia. Il Radiologo 2006;2:97-100.

3. Fryback D, Thornbury JR. The efficacy of diagnostic imaging. Med Decis Making 1991;11:88-94.

4. Thornbury JR. Clinical efficacy of diagnostic imaging: love it or leave it, AJR Am J Roentgenol 1994;162:1-8.

5. Cochrane A. Efficacia ed efficienza in medicina. Roma: Il Pensiero Scientifico; 2000.

6. Smith TJ. Evidence-based follow-up of lung cancer patients. Semin Oncol 2003;30:361-8.

7. Bosisio E. Il governo clinico. In: Sanità pubblica e privata. Santarcangelo di Romagna (RN): Maggioli Editore; 2005. pp 60-67.
8. Bono A, Campanini A. The METIS project for generalist ultrasonography. J Ultrasound 2009;XX:1e7.

9. Mechi MT, Ricci D, Petrillo M, et al. Progetto Regione Toscana "NET-VISUAL DEA" per l'ottimizzazione del flusso pazienti dal pronto soccorso ai reparti di degenza. In: $7^{\circ}$ Conferenza Nazionale GIMBE. Evidence \& Governance per la sostenibilità della sanità pubblica; Bologna, 17 febbraio 2012; Abstract No. 96. Available from: gimbe.org/conferenze/nazionale/2012/Abstract_book.pdf

10. Scalea TM, Rodriguez A, Chiu WC, et al. Focused assessment with sonography for trauma (FAST): results from an international consensus conference. J Trauma 1999;46:466-72.

11. Rozycki GS, Shackford SR. Ultrasound, what every trauma should know. J Trauma 1996;40:1-4.

12. Blackbourne LH, Soffer D, McKenney M, et al. Secondary ultrasound examination increases the sensitivity of the FAST exam in blunt trauma. J Trauma 2004;57:934-8.

13. Gaarder C, Kroepelien CF, Loekke R, et al. Ultrasound performed by radiologists-confirming the truth. J Trauma 2009;67:323-9.

14. American College of Radiology. Appropriateness criteria for imaging and treatment decisions. Reston, VA: American College of Radiology; 1995.

15. Kohn LT, Corrigan JM, Donaldson MS. To err is human: building a safer Health System, Insitute of Medicine. Washington, DC: National academy Press; 1999.

16. Berner ES, Graber ML. Overconfidence as a cause of diagnostic error in medicine, Am J Med 2008;121:S2-23.

17. Croskerry P, Norman G. Overconfidence in clinical decision making. Am J Med 2008;121:S24-9.

18. Ashmann CJ, Yu JS, Wolfman D. Satisfaction of search in osteoradiology. AJR 2000;175:541-4.

19. Pinto A, Brunese L. Spectrum of diagnostic errors in radiology. World J Radiol 2010;2:377-83.

20. Schiff GD. Minimizing diagnostic error: the importance of follow-up and feedback. Am J Med 2008;121:S38-42.

21. European Society of Radiology (ESR). European Commission guidelines on clinical audit. Statement by the European Society of Radiology. Insights Imaging 2011;2:97-8.

22. Wright J, Hill P. La governance clinica. Milano: McGraw-Hill; 2005. 